\title{
Early Marjolin's ulcer developing in a penile human bite scar of an adult patient presenting at Bugando Medical Centre, Tanzania: A case report
}

\author{
PHILLIPO L. CHALYA ${ }^{1 *}$, JOSEPH B. MABULA ${ }^{1}$, JAPHET M. GILYOMA ${ }^{1}$, PETER RAMBAU ${ }^{2}$, NESTORY \\ MASALU $^{3}$ and SAMSON SIMBILA ${ }^{1}$ \\ ${ }^{1}$ Department of Surgery, Catholic University of Health and Allied Sciences- Bugando, Mwanza, Tanzania \\ ${ }^{2}$ Department of Pathology, Catholic University of Health and Allied Sciences- Bugando, Mwanza, Tanzania \\ ${ }^{3}$ Department of Oncology, Catholic University of Health and Allied Sciences- Bugando, Mwanza, Tanzania
}

\begin{abstract}
Marjolin's ulcer is a rare but well documented cutaneous malignancy that arises in a scar or chronic ulcer, and is characterized by an aggressive course. The latent period from the injury to the appearance of cancer has been reported to be 25-40 years. Early occurring Marjolin's ulcer has rarely been described in the literature and its development in a penile human bite scar is still rare. We report a rare case of a 33 year-old patient who presented with an early appearance of Marjolin's ulcer developing in a penile human bite scar. On histological examination it was found to be poorly differentiated squamous cell carcinoma. He was managed by total penile amputation. Five months after surgery, the patient presented to our surgical outpatient clinic with inguinal lymph node metastases and distant metastases to the lung, liver and bones. However, the patient died shortly after admission. This report highlights the possibility of early appearance of Marjolin's ulcer arising from a chronic ulcer or healed scar in an unusual sites such as the penis. It is therefore recommended that all chronic ulcers should be thoroughly investigated at presentation, to avoid labelling malignancies 'chronic ulcers', leading to delay in appropriate treatment. Early recognition and aggressive treatment of Marjolin's ulcers and close followup are urgently needed to improve outcomes in our environment.
\end{abstract}

Keywords: Marjolin's ulcer, malignant transformation, penis, human bite, Tanzania

\section{Introduction}

Marjolin's ulcer is a rare and often aggressive cutaneous malignancy that arises in previously traumatized or chronically inflamed skin, particularly after burns (Sabin et al, 2004). In 1828, Jean-Nicholas Marjolin, a French surgeon described the occurrence of tumours in posttraumatic scar tissue but did not recognize its malignant nature (Treves \& Pack, 1930). However, his name is now synonymous with a variety of cancers arising in any scar tissue or chronic wounds. The reason for malignant transformation is largely unknown (Er-Fan et al, 1992, Thio et al, 2003).

The commonest type of carcinoma arising from Marjolin's ulcer is squamous cell carcinoma, followed by basal cell carcinoma as the second commonest carcinoma (Suhag et al, 2005).The squamous cell carcinoma of Marjolin's ulcer has the worst prognosis in comparison with other squamous cell carcinomas and this requires an aggressive treatment (Ahmad, 2009).

Most lesions of Marjolin's ulcer occur on the extremities, ulcers on the head and face occurs less frequently and those on the trunk least frequent (Treves \& Pack, 1930). The latent period between the injury and the appearance of cancer is 25-40 years (Malheiro et al, 2001).

\footnotetext{
* Correspondence to: Phillipo Leo Chalya; E-mail: drphillipoleo@yahoo.com
} 
However, early occurring Marjolin's ulcer has also been described in the literature (Çeliköz et al, 1997, Thio et al, 2003). It has been reported that the patient age at the time of injury influences latency time, which is inversely proportional to patient's age at the time of injury (Lawrence, 1952). However, Thio et al (2003 have reported malignant transformation within 18 months in lower limb wounds. Soto-Davalos et al (2008) have reported malignant transformation as early as three months. In this report, we present a rare case of an early appearance of Marjolin's ulcer developing in a penile human bite scar. To the best our knowledge this patient may be the first reported case in literature presenting with early penile Marjolin's ulcer developed in a human bite scar.

\section{Case report}

A 33-year- old Tanzanian male with no co-morbidities presented to our surgical out-patient clinic with a 4-month history of a non-healing, fungating ulcer involving the entire two-third of the penile shaft. He reported to have sustained human bite injury on his glans and distal part of the penile shaft seven months prior to the onset of this ulcer. He reported to have sustained penile human bite injury during oral sexual intercourse, the mechanism of which could not be established. He was managed at Shinyanga Regional Hospital by surgical debridement and primary closure of the skin. The wound healed satisfactorily after two weeks of treatment and follow up at Shinyanga Regional Hospital.

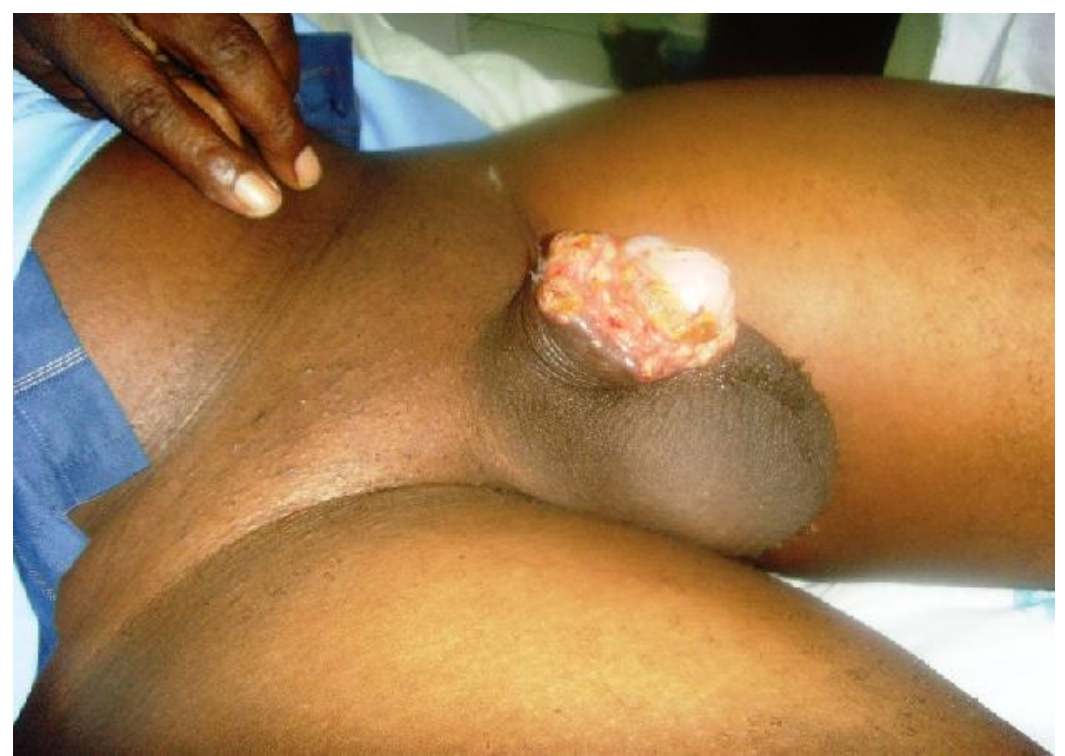

Figure 1: A 33 year-old male patient presenting with a penile Marjolin's ulcer developed in a human bite scar, seven months after the initial injury

Seven months after the injury he developed an unremitting ulcer at the site of a previous healed scar. The ulcer was very small to begin with but later on increased in size rapidly with easy bleeding on touch and foul smelling mucopurulent discharge. He attended at Kolandoto Mission Hospital where he was treated as a case of syphilitic ulcer with no improvement and he was later referred to our centre for expertise management. However, instead of reporting to our 
tertiary care hospital, he first went to traditional healers where he was treated for two months with no improvement. There was no history of diabetes, hypertension or tuberculosis.

At presentation he was healthy looking, not wasted, not anaemic, not dyspnoeic and he was not icteric. There was an almost circumferential non-healing, fungating ulcer involving almost the entire circumference of the two-third of the penile shaft. The edges were raised, with foul-smelling purulent discharge from the floor, which was dirty with slough and unhealthy granulation tissues (Figure 1). The inguinal lymph nodes were not palpable. Systemic examination revealed no other pathologies.

Complete hemogram, blood biochemistry, chest x-ray and abdominal ultrasound were essentially normal. The patient underwent incisional biopsy of the ulcerated lesion. Histopathological examination of the specimen showed invasive poorly differentiated squamous cell carcinoma. A diagnosis of penile cancer stage II was made.

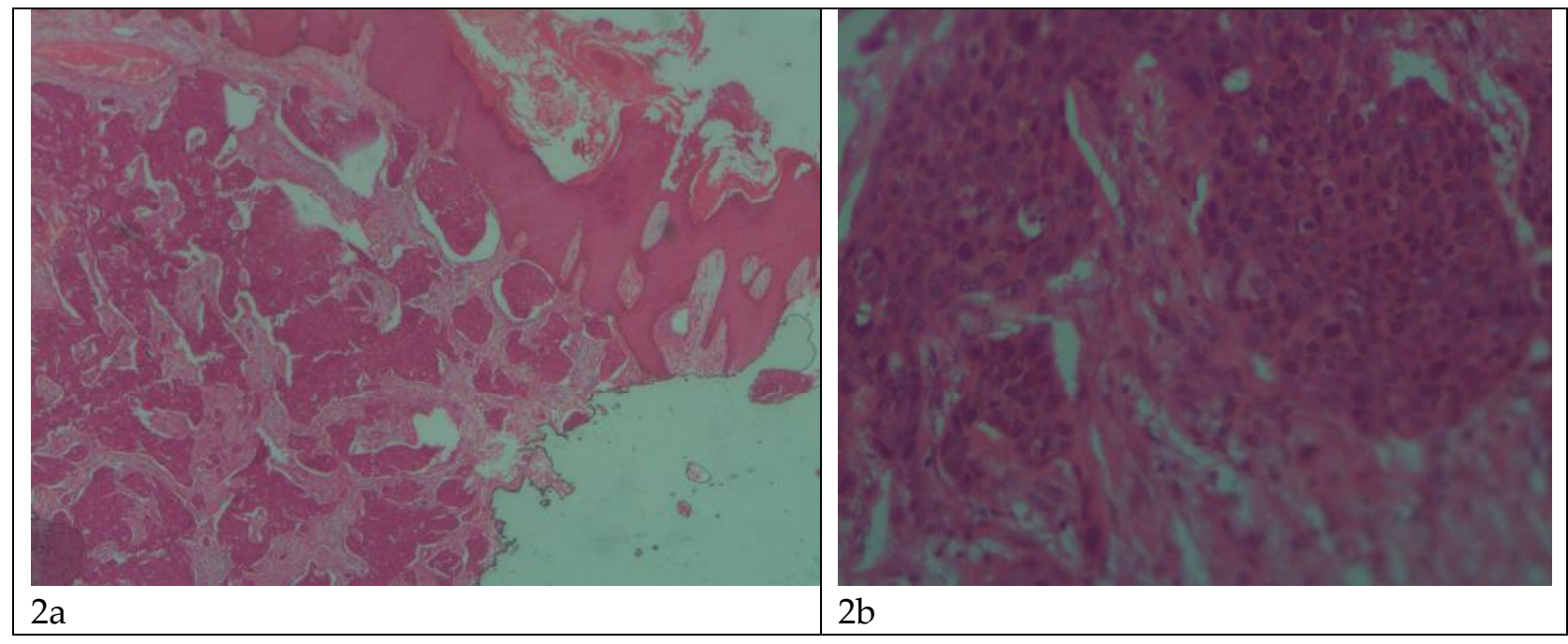

Figure 2a: Histology showing (2a) verucoid penile skin with underlying infiltrative squamous cell carcinoma (x5) (2a) and malignant invasive poorly differentiated squamous cell carcinoma with large pleomorphic hyperchromatic cells (x 20) (2b)

After confirmation of the diagnosis, the patient was prepared for definitive surgery and underwent total penile amputation. Histopathological examination of the amputated lesion revealed a poorly differentiated squamous cell carcinoma with resected margins microscopically free from the tumour (Figure 2a, b). Postoperatively the patient made an uneventful recovery and was discharged on the tenth postoperative day advised to be seen at our surgical outpatient clinic on regular basis for follow up. However, the patient lost to follow up after attending only once at three month post-surgery.

Five months after his definitive surgery, he was seen at our surgical outpatient clinic with difficulty in breathing, lower backache and nodular hepatomegally. He had tinge of jaundice and right inguinal lymph node enlargement Chest and lumbosacral spines radiographs and abdominal ultrasound revealed lung, bone and liver metastases. He had no local tumour recurrence. He was referred to Ocean Road Cancer Institute (ORCI) for possible 
palliative therapy. However the patient died shortly after admission before receiving any palliative therapy.

\section{Discussion}

Marjolin's ulcer is defined as a tumour arising from a chronically inflamed, traumatized or scarred area of the skin (Sabin et al., 2004). It is a rare clinical entity and commonly these lesions are treated as chronic ulcers and consequently can be easily missed (Hahn et al., 1990). This observation is reflected in our patient as he was initially treated in the peripheral hospitals as a case of syphilitic ulcer with no improvement. A suspicion of malignant change should be entertained in the presence of crusting, ulceration of scar tissue, increase in pain or size of the ulcer, and bleeding (Thio et al., 2003). Delayed diagnosis may result in the need for more extensive surgery or increase the risk of metastatic spread.

Jean-Nicholas Marjolin originally described the malignant transformation of cutaneous scars in 1828 (Daya \& Balakrishan, 2009). Marjolin's ulcers have a 1\% to 2\% incidence in all burn scars but can also develop from previously traumatized and scarred tissue of other aetiologies such as chronic osteomyelitis, post-traumatic wounds and chronic fistulae (Treves \& Pack, 1930). Our patient developed Marjolin's ulcer from post-traumatic (penile human bite) scar in an unusual site.

The exact mechanism of malignant transformation is largely unknown, and many theories have been postulated. Early theories suggested that cellular mutations as a result of inflammatory related substances release by damaged, ischemic and nutritionally deficient tissues are responsible for neoplastic change (Treves \& Pack, 1930). Neuman et al. (1963) proposed that traumatic displacement of living epithelial tissue into dermis may cause a foreign body response and lead to a deranged regenerative process, resulting in carcinomatous change. More recently, a theory of immunologic isolation has been suggested, whereby lymphatic channel obliteration at the site of injury decreases the delivery of antigen or specifically stimulated small lymphocytes to the regional lymphocytes from that site. This renders the site 'immulogically unprivileged', allowing the development of antigenically foreign tumour cells to go unchecked. Such cells may initially arise by spontaneous mutation or develop under the influence of viral or chemical carcinogens. Tumour antigen recognition may then be delayed long enough for tumours to reach 'critical size', when immune mechanisms are no longer sufficient to prevent continued neoplastic progression (Bostwitch et al., 1975). It has also been suggested that patients with an inherent immune deficiency are at higher risk for developing malignant ulcers (Trent \& Kirsner, 2003). Some authors have also postulated that with chronic irritation and repeated damage of the ulcer, there is continuous mitotic activity as the epidermal cells attempt to resurface the open defect. This cycle of damage, irritation, and repair can lead to a malignant transformation (Copcu et al., 2003). More recent theories have included genetic postulations involving human leukocyte antigen (HLA) DR4 and mutations in the p53 and/or Fas genes (Lee et al., 2000; Hayashi et al., 2003).

The latent period between original injury and development of Marjolin's ulcer has been document as being as long as 30 years (Hahn et al., 1990). Our patient in this study presented with an early appearance of Marjolin's ulcer that developed in a penile human bite scar only 
seven months following the initial injury. In a literature search, we could not find any instances of early appearance of Marjolin's ulcer in a penile human bite scar.

Marjolin's ulcer has been reported to occur in $40 \%$ of cases in the lower extremities, 30\% in the head and neck region, 20\% in the upper extremities, and 10\% in the trunk (Lawrence, 1952, Novick et al, 1977, Hill et al, 1996). They have also been documented in unusual sites such as the genitalia as a complication of Fournier's gangrene (Chintamani et al, 2004) and breast skin developing in a post-burn scar (Antonino et al., 2008). The reason for this anatomical site predilection is not well understood. Our patient developed Marjolin's ulcer in a penile human bite scar. We found no report in literature search that demonstrated Marjolin's ulcer in the penis reflecting the rarity of this cancer in this anatomical site.

The commonest type of carcinoma arising from Marjolin's ulcer is squamous cell carcinoma, followed by basal cell carcinoma as the second commonest carcinoma (Suhag et al., 2005). Other reported neoplasms are malignant melanoma, osteogenic sarcoma, fibrosarcoma and liposarcoma (Thio et al., 2003). As expected the histopathological examination of the specimen in our patient showed poorly differentiated squamous cell carcinoma.

Marjolin's ulcers have been reported to have an aggressive course and a much greater tendency to metastasize than squamous cell carcinomas arising from other causes, which makes early diagnosis imperative (Chintamani et al., 2004; Suhag et al., 2005; Antonino et al., 2008; Chalya et al., 2012). Metastasis to the brain, liver, lung, kidney, and distant lymph nodes have been commonly reported (Treves \& Pack, 1930). Our patient in this study had inguinal lymph node metastases and distant metastases to the lung, liver and bones, five months after definitive surgery. Our patient lost to follow up following definitive surgery and he could not receive any adjuvant therapy. This observation calls for emphasis on long-term follow up after definitive surgery to be able to detect and manage metastases at an early stage. Sentinel lymph node biopsy is highly recommended to be able to detect occult nodal involvement at early stage of the disease. However, sentinel lymph node biopsy is not done in our setting.

In conclusion, this report highlights the possibility of early appearance of Marjolin's ulcer arising from a chronic ulcer or healed scar in an unusual sites such as the penis. It is therefore recommended that all chronic ulcers should be thoroughly investigated at presentation, to avoid labelling malignancies 'chronic ulcers', leading to delay in appropriate treatment. Health education is needed to discourage patients with cutaneous ulcers from presenting to traditional healers and chemists. Education is needed as well to prevent late presentation. Early adequate treatment of all ulcers and scars will certainly reduce the incidence of Marjolin's ulcer. Aggressive treatment and close long-term follow-up are urgently needed to improve outcomes in our environment.

\section{Consent}

Written informed consent was obtained from the patient's relatives for publication of this case report and accompanying images. 


\section{References}

Ahmad, S. (2009) An Overview of Heel Marjolin's Ulcers in the Orthopedic Department of Urmia University of Medical Sciences. Archives of Iranian Medicine 12, $405-408$.

Antonino, G., Simone, S., Laura, F., Christina, G. \& Claudio, L. (2008) A rare case of aggressive squamous-cell carcinoma of the breast skin: Marjolin's ulcer. Chirurgia Italiana 60, 577582.

Bostwick, J., Pendergrast, W.J. \& Vasconez V. (1975) Marjolin's ulcer: an immunologically privileged tumor? Plastic and Reconstructive Surgery 57, 66-9

Çeliköz, B., Demiriz, M. \& Selmanpakolu, N. (1997) A shorter lag period of mesenchymal malignancy in Marjolin's ulcer. Burns 23, 72-4

Chalya, P.L., Mabula, J.B., Rambau, P., Mchembe, D.M., Kahima, J.K., Chandika, A.B., Giiti, G., Masalu, N., Ssentongo, R. \& Gilyoma, J.M. (2012) Marjolin's ulcers at a university teaching hospital in Northwestern Tanzania: a retrospective review of 56 cases. World Journal of Surgical Oncology 10, 38.

Chintamani, M., Shankar, M., Singhal, V., Singh, J.P., Bansal, A. \& Saxena, S. (2004) Squamous cell carcinoma developing in the scar of Fournier's gangrene Case report. BMC Cancer27, 16.

Copcu, E., Aktas, A., Sismant, N. \& Oztan, Y. (2003) Thirty-one cases of Marjolin's ulcer. Clinical and Experimental Dermatology 28, 138-41.

Daya, M. \& Balakrishan, T. (2009) Advanced Marjolin's ulcer of the scalp in a 13-year-old boy treated by excision and free tissue transfer: Case report and review of literature. Indian Journal of Plastic Surgery 42, 106-111.

Er-Fan, X., Li, A.O., Shi-ling, W., Shao-Yu, K. \& Guang-Xiu, C. (1992) Burn scar carcinoma: Case reports and review of the literature. Annals of the $M B C 5,2$

Hahn, S.B., Kim, D.J. \& Jeon, C.H. (1990) Clinical study of Marjolins ulcer. Yonsei Medical Journal 31, 234-241.

Hayashi, M., Tamura, G., Kato, N., Ansai, S., Kondo, S. \& Motoyama, T. (2003) Genetic analysis of cutaneous squamous cell carcinomas arising from different areas. Pathology International 53, 602-607.

Hill, B.B., Sloan, D.A., Lee, E.Y., McGrath, P.C. \& Kenady, D.E. (1996) Marjolin's ulcer of the foot caused by non-burn trauma. Southern Medical Journal 89,707-710.

Lawrence, E. (1952) Carcinoma arising in the scars of thermal burns: with special reference to the influence of the age at burn on the length of the induction period. Surgery, Gynecology E Obstetrics 95, 579 - 588.

Lee, S.H., Shin, M.S. \& Kim, H.S. (2000) Somatic mutations of Fas (Apo-1/CD95) gene in cutaneous cell carcinomas arising from a burn scar. Journal of Investigative Dermatology 114, 122-126.

Malheiro, E., Pinto, A. \& Choupina, M. (2001) Marjolin's ulcer of the scalp: Case report and literature review. Annals of Burns and Fire Disasters 14, 115-118.

Neuman, Z., Ben-Hur, N. \& Shulman, J. (1963) Trauma and skin cancer. Implantation of epidermal elements as possible cause. Plastic and Reconstructive Surgery 32, 649-656. 
Novick, M., Gard, D.A. \& Hardy, S.B. (1977) Burn scar carcinoma: Review and analysis of 46 cases. Journal of Trauma 17, 809-817.

Sabin, S.R., Goldstein, G., Rosenthal, H.G. \& Haynes, K.K. (2004) Aggressive squamous cell carcinoma originating as a Marjolin's ulcer. Dermatologic Surgery 30, 229-230.

Soto-Dávalos, B.A., Cortés-Flores, A.O. \& Bandera-Delgado, A. (2008) Malignant neoplasm in burn scar: Marjolin's ulcer. Report of two cases and review of the literature Cir Cir 76, 329-331

Suhag, V., Singh, S., Nimbrian, VK., Dimri, K., Sharma, N. (2005) Marjolin's Ulcer developing in electrical burns: A rare case report. Pakistan Journal of Medical Science 21, 375-6

Thio, D., Clarkson, J.H., Misra, A. \& Srivastava, S. (2003)Malignant change after 18 months in a lower limb ulcer: Acute Marjolin's ulcer revisited. British Journal of Plastic Surgery 56, 825-8

Trent, J.T. \& Kirsner, RS. (2003) Wounds and malignancy. Advances in Skin and Wound Care 16, $31-34$

Treves, N. \& Pack, GT. (1930) Development of cancer in burn scars. Surgery, Gynecology $\mathcal{E}$ Obstetrics 512, 749-82 